\title{
LEVELS AND SUBLEVELS OF QUATERNION ALGEBRAS
}

\author{
DETLEV W. HOFFMANN
}

Dedicated to Professor David W. Lewis on the occasion of his 65th birthday

\begin{abstract}
The level $s$ (resp. sublevel $\underline{s}$ ) of a ring $R$ with $1 \neq 0$ is the smallest positive integer such that -1 (resp. 0 ) can be written as a sum of $s$ (resp. $\underline{s}+1$ ) nonzero squares in $R$, provided -1 (resp. 0 ) is a sum of nonzero squares at all. D.W. Lewis showed that any value of type $2^{n}$ or $2^{n}+1$ can be realized as level of a quaternion division algebra, and in all these examples, the sublevel was $2^{n}$, which prompted the question whether or not the level and sublevel of a quaternion division algebra will always differ at most by one. In this note, we give a positive answer to that question.
\end{abstract}

\section{IntRoduction}

Let $D$ be a division ring. The level $s(D)$ and the sublevel $\underline{s}(D)$ of $D$ are defined as follows:

(1) If -1 is a sum of squares in $D$, then

$$
s(D)=\min \left\{n \mid \exists x_{1}, \ldots, x_{n} \in D:-1=x_{1}^{2}+\ldots+x_{n}^{2}\right\} .
$$

Otherwise, $s(D)=\infty$.

(2) If 0 is a sum of nonzero squares in $D$, then

$$
\underline{s}(D)=\min \left\{n \mid \exists x_{1}, \ldots, x_{n+1} \in D^{*}=D \backslash\{0\}: 0=x_{1}^{2}+\ldots+x_{n+1}^{2}\right\} .
$$

Otherwise, $\underline{s}(D)=\infty$.

It is clear from the definition that $\underline{s}(D) \leq s(D)$, and one readily sees that if $D$ is a (commutative) field, the $s(D)=\underline{s}(D)$.

The study of level and sublevel of rings has a history dating back at least to the early 20th century. A famous result by Pfister [9] states that the level of a field, if finite, is always a 2-power, and that each 2-power can be realized as level of a field. This answered a question posed by Van der Waerden in the 1930s.

The study of levels and sublevels in the above sense for noncommutative division rings started in the mid-1980s. In [5], [6], David Lewis showed that for every $k \in \mathbb{N}$, there exist quaternion division algebras with $s=\underline{s}=2^{k}$ and with $s=\underline{s}+1=2^{k}+1$, and that for any quaternion division algebra $D$ with $s(D)=2^{k}$ one also has $\underline{s}(D)=$ $2^{k}$. Leep [4] gave slight improvements on some of Lewis's results, and he asked the following questions (already implicit in [5], [6] and reiterated in [7]):

The idea for this paper came during a conference at University College Dublin held on the occasion of the 65th birthday of Professor David Lewis. The author thanks UCD and in particular Thomas Unger for their hospitality. 
Question. (1) Can the level (resp. sublevel) of a quaternion division algebra $D$ take values that are not of the form $2^{k}, 2^{k}+1$ (resp. $\left.2^{k}\right)$ ?

(2) Does one always have $s(D) \leq \underline{s}(D)+1$ ?

As for the first question, quaternion division algebras of sublevel 3 were constructed by Krüskemper and Wadsworth [2]. It was shown in [1] that for each $k \geq 2$, there exist quaternion division algebras $D$ with $2^{k}+2 \leq s(D) \leq 2^{k+1}-1$ (although the method used there to construct such $D$ by employing function fields of quadrics does not allow to give the exact value for $s(D))$. O'Shea [8] observed that this function field method also allows to construct quaternion division algebras $D$ of sublevel not of the form $2^{k}$ and $>3$. It is still not fully known what exact values can be realized as (sub)levels of quaternion division algebras.

In this note, we give a positive answer to the second question:

Theorem. Let $D$ be a quaternion division algebra. Then $\underline{s}(D) \leq s(D) \leq \underline{s}(D)+1$.

\section{Proof of the Theorem}

We first recall a few simple facts about quaternion algebras. We refer to [3, chapter III] for any facts we use without further reference.

Let $F$ be a field of characteristic different from 2 and let $D=(a, b)_{F}\left(a, b \in F^{*}\right)$ be the quaternion algebra with $F$-basis $\{1, i, j, k\}$ subject to the relations $i^{2}=a$, $j^{2}=b, i j=-j i=k$. We assume $D$ to be a division algebra, which is equivalent to saying that its norm form $\langle 1,-a,-b, a b\rangle$ is anisotropic.

For $\zeta=x+y i+z j+w k \in D(x, y, z, w \in F)$, we call $x$ the scalar part of $\zeta$, and $\zeta^{\prime}=y i+z j+w k$ its pure part. We put $D^{\prime}=F i+F j+F k$, the subspace of pure quaternions. We have $\zeta^{2}=x^{2}+2 x \zeta^{\prime}+\zeta^{\prime 2}$ with $\zeta^{\prime 2}=a y^{2}+b z^{2}-a b w^{2} \in F$. The quadratic form $\langle a, b,-a b\rangle$ will be denoted by $T_{P}$. We immediately get the following well known lemma:

Lemma. $c \in F$ is a sum of $m$ squares of pure quaternions in $D$ (not all squares equal to 0 if $c=0$ ) if and only if the quadratic form

$$
m \times T_{P}=\underbrace{T_{P} \perp \ldots \perp T_{P}}_{m}
$$

represents $c$ (nontrivially if $c=0$, i.e. $m \times T_{P}$ is isotropic in that case).

Proof of the Theorem. Let $D$ be a quaternion division algebra as above and assume that $\underline{s}(D)=m$. We only have to show that $s(D) \leq m+1$. Let $\zeta_{\ell} \in D^{*}, 1 \leq \ell \leq$ $m+1$ be such that

$$
0=\zeta_{1}^{2}+\ldots+\zeta_{m+1}^{2} .
$$

Write $\zeta_{\ell}=x_{\ell}+\zeta_{\ell}^{\prime}$ with $x_{\ell} \in F$ and $\zeta_{\ell}^{\prime} \in D^{\prime}$. We get

$$
0=\sum_{\ell=1}^{m+1} x_{\ell}^{2}+2 x_{\ell} \zeta_{\ell}^{\prime}+\zeta_{\ell}^{\prime 2}
$$

and thus

$$
\sum_{\ell=1}^{m+1} x_{\ell}^{2}+\zeta_{\ell}^{\prime 2}=0=\sum_{\ell=1}^{m+1} x_{\ell} \zeta_{\ell}^{\prime}
$$

1. case: All $x_{\ell}=0,1 \leq \ell \leq m+1$.

In this case, 0 is a nontrivial sum of squares of $m+1$ pure quaternions, so $(m+1) \times T_{P}$ 
is isotropic by the Lemma. But then $(m+1) \times T_{P}$ contains a hyperbolic plane $\langle 1,-1\rangle$ as subform, in particular, $(m+1) \times T_{P}$ represents -1 . Again by the Lemma, we have that -1 is a sum of squares of $m+1$ pure quaternions, hence $s(D) \leq m+1$. 2. case: $\sum_{\ell=1}^{m+1} x_{\ell}^{2}=0$ but not all $x_{\ell}=0$.

In this case, 0 is a nontrivial sum of $m+1$ squares already in $F$, and thus $s(D) \leq$ $s(F)=\underline{s}(F) \leq m$.

3. case: $\sum_{\ell=1}^{m+1} x_{\ell}^{2} \neq 0$.

Let

We then get

$$
c_{\ell}=\frac{x_{\ell}}{x_{1}^{2}+\cdots+x_{m+1}^{2}}
$$

$$
\sum_{\ell=1}^{m+1} c_{\ell} \zeta_{\ell}=\frac{1}{x_{1}^{2}+\ldots+x_{m+1}^{2}}(\sum_{\ell=1}^{m+1} x_{\ell}^{2}+\underbrace{\sum_{\ell=1}^{m+1} x_{\ell} \zeta_{\ell}^{\prime}}_{=0})=1 .
$$

Put $c=c_{1}^{2}+\ldots+c_{m+1}^{2}=\left(x_{1}^{2}+\ldots+x_{m+1}^{2}\right)^{-1}$. This yields

$$
\begin{aligned}
\sum_{\ell=1}^{m+1}\left[\left(\frac{c+1}{2}\right) \zeta_{\ell}-c_{\ell}\right]^{2} & =\left(\frac{c+1}{2}\right)^{2} \underbrace{\sum_{\ell=1}^{m+1} \zeta_{\ell}^{2}}_{=0}-(c+1) \underbrace{\sum_{\ell=1}^{m+1} c_{\ell} \zeta_{\ell}}_{=1}+\underbrace{\sum_{\ell=1}^{m+1} c_{\ell}^{2}}_{=c} \\
& =-1,
\end{aligned}
$$

which shows that $s(D) \leq m+1$.

Remark. The above proof can be used more or less verbatim in the case of octonion division algebras (with the appropriate notions of pure octonion and of the form $T_{P}$ corresponding to squares of pure octonions). So if $\mathcal{O}$ is an octonion division algebra, one also gets that $\underline{s}(\mathcal{O}) \leq s(\mathcal{O}) \leq \underline{s}(\mathcal{O})+1$.

\section{REFERENCES}

[1] D.W. Hoffmann, Levels of quaternion algebras, Arch. Math. (Basel) 90 (2008), 401-411.

[2] M. Krüskemper and A.R. Wadsworth, A quaternion algebra of sublevel 3, Bull. Soc. Math. Belg. Sér. B 43 (1991), 181-185.

[3] T.Y. Lam, Introduction to quadratic forms over fields, Graduate Studies in Mathematics 67, American Mathematical Society, Providence, RI, 2005.

[4] D.B. Leep, Levels of division algebras, Glasgow Math. J. 32 (1990), 365-370.

[5] D.W. Lewis, Levels and sublevels of division algebras, Proc. Roy. Irish Acad. Sect. A 87 (1987), 103-106.

[6] D.W. Lewis, Levels of quaternion algebras, Quadratic forms and real algebraic geometry (Corvallis, OR, 1986). Rocky Mountain J. Math. 19 (1989), 787-792.

[7] D.W. Lewis, Levels of fields and quaternion algebras - a survey, Théorie des nombres, Années 1996/97-1997/98, Publ. Math. UFR Sci. Tech. Besançon, Univ. Franche-Comté, Besançon, 1999, 9 pp.

[8] J. O'Shea, Bounds on the levels of composition algebras, Math. Proc. R. Ir. Acad. (to appear).

[9] A. Pfister, Zur Darstellung von -1 als Summe von Quadraten in einem Körper, J. London Math. Soc. 40 (1965), 159-165.

School of Mathematical Sciences, University of Nottingham, University Park, NotTINGHAM NG7 2RD, UK

E-mail address: detlev.hoffmann@nottingham.ac.uk 